\title{
Discussion on Electrical Performance Design of Broadband Radome
}

\author{
Qiong Li
}

Yunnan Traffic and Communication School , Kunming, Yunnan, China, 650504

Keywords: Broadband, Radome, Electrical Performance Design

\begin{abstract}
Conventional radome bandwidth is narrow, band bandwidth usually refers to the center frequency of $0.85 \%$, it is difficult to meet the needs of anti-radiation seeker system, so that the radome wide band difficult to meet its requirements. In this paper, the design method of electrical performance of wideband radome and its related design principle are discussed and analyzed. The design of the equivalent network of wideband radome is introduced, and the relevant design examples are put forward to provide reference and reference for related design fields.
\end{abstract}

\section{Introduction}

Broadband radome is a key technology in all kinds of wideband seeker. In order to meet the requirements of high-speed flight and precision guidance, the broadband radome needs to have a certain strength, and has high temperature, high power transmission, aiming line low error slope and low error line and other characteristics [1]. At present, Chinese wide-band radome technology research is not a lot, and broadband radome technology and its electrical performance design research is also very urgent to promote the development of broadband seeker.

\section{Research on Broadband Radome Design Theory}

The design theory of wideband radome is based on the theory of microwave transmission line. At the end of 20c30s, some scholars have simulated the efficiency of propagation of wavelengths in dielectric medium and propagation in long lines, and the theory of dielectric wave impedance from the point of view of the plane wave, the wave impedance where $\mu$ and $\varepsilon$ are the permeability and the dielectric constant, respectively. According to the comparison between the wave impedance type and the wavelength in the dielectric medium and the long line, two kinds of dielectrics the characteristics of media boundary conditions are different [2]. In the case of oblique incidence on the boundary, the expression of the wave impedance can be pushed according to the electromagnetic theory, and two kinds of medium with infinity can be used to derive the wave impedance expression of parallel polarization and vertical polarization.

$$
\begin{gathered}
Z / / 1=\sqrt{\frac{\mu_{1}}{\varepsilon_{1}}} \cos \theta_{1} \quad Z / / 2=\sqrt{\frac{\mu_{2}}{\varepsilon_{2}}} \cos \theta_{2} \\
Z \perp_{1}=\sqrt{\frac{\mu_{1}}{\varepsilon_{1}} \frac{1}{\cos \theta_{1}} \quad Z \perp_{2}=\sqrt{\frac{\mu_{2}}{\varepsilon_{2}}} \frac{1}{\cos \theta_{2}}}
\end{gathered}
$$

Where $\mathrm{Z} / / \mathrm{i}$ and $\mathrm{Z} \perp \mathrm{i}$ are the waveguides of horizontal polarization and vertical polarization, and the dielectric constants in the two different dielectrics are given by $i=1,2,3, \ldots, n ; \varepsilon 1, \mu 1$ and $\varepsilon 2, \mu 2$, and the incident angle of the electromagnetic wave to the two media is represented by $\theta 1$ and $\theta 2$, respectively [3].

In the case where both the wave impedance and the medium wavelength are known, it can be found that the input impedance of the medium segment with length $d$ is based on the simulation of the wavelength in the long line and the dielectric medium.

$$
Z_{d}=\frac{E_{x}}{H_{y}}=Z_{1 \theta} \frac{Z_{2 \theta} \cos \beta_{1} d+j Z_{1 \theta} \sin \beta_{1} d}{Z_{1 \theta} \cos \beta_{1} d+j Z_{2 \theta} \sin \beta_{1} d}
$$


Electromagnetic wave in the dielectric medium $1, \beta 1$ is a constant that the electromagnetic wave propagates along the normal direction of the dielectric boundary. Electromagnetic waves in the transmission is along the air to the media, and then from the media to the direction of the air to spread in the process of propagation through different boundaries, this process and multi-layer dielectric wall equivalent circuit equivalent effect, its essence The impedance has changed [4].

According to formula 1 and formula 2, it can be deduced that the parallel polarization and vertical polarization normalized impedance formulas can be expressed as:

$$
\left\{\begin{array} { l } 
{ Z / / 0 = \sqrt { 1 - \operatorname { s i n } ^ { 2 } \theta } } \\
{ Z / / 1 = \frac { 1 } { \varepsilon _ { 1 } } \sqrt { \varepsilon _ { 1 } - \operatorname { s i n } ^ { 2 } \theta } } \\
{ \cdots \cdots } \\
{ Z / / n = \frac { 1 } { \varepsilon _ { n } } \sqrt { \varepsilon _ { n } - \operatorname { s i n } ^ { 2 } \theta } }
\end{array} \left\{\begin{array}{l}
Z \perp_{\perp}=\frac{1}{\sqrt{1-\sin ^{2} \theta}} \\
\ldots \ldots \\
Z \perp_{\perp n}=\frac{1}{\sqrt{\varepsilon_{1}-\sin ^{2} \theta}} \\
\sqrt{\varepsilon_{n}-\sin ^{2} \theta}
\end{array}\right.\right.
$$

In the case of polarization, the radome of the cover wall of the same thickness of the case of its expression:

$$
\left\{\begin{array}{l}
\varphi_{1}=\frac{2 \pi}{\lambda} d_{1} \sqrt{\varepsilon_{1}-\sin ^{2} \theta} \\
\varphi_{2}=\frac{2 \pi}{\lambda} d_{2} \sqrt{\varepsilon_{2}-\sin ^{2} \theta} \\
\cdots \cdots \\
\varphi_{n}=\frac{2 \pi}{\lambda} d_{n} \sqrt{\varepsilon_{n}-\sin ^{2} \theta}
\end{array}\right.
$$

The equivalent impedance of each layer of parallel polarization and vertical polarization is expressed by $\mathrm{Z} / / 0, \mathrm{Z} / / 1, \ldots, \mathrm{Z} / / \mathrm{n}$ and $\mathrm{Z} \perp 0, \mathrm{Z} \perp 1, \ldots, \mathrm{Z} \perp \mathrm{n}$, and each layer The electrical constant is expressed by $\varepsilon 1, \ldots$, $\varepsilon n$ [5]. The graph of the equivalent impedance with the change of the dielectric constant and the incident angle is shown in Fig 1.
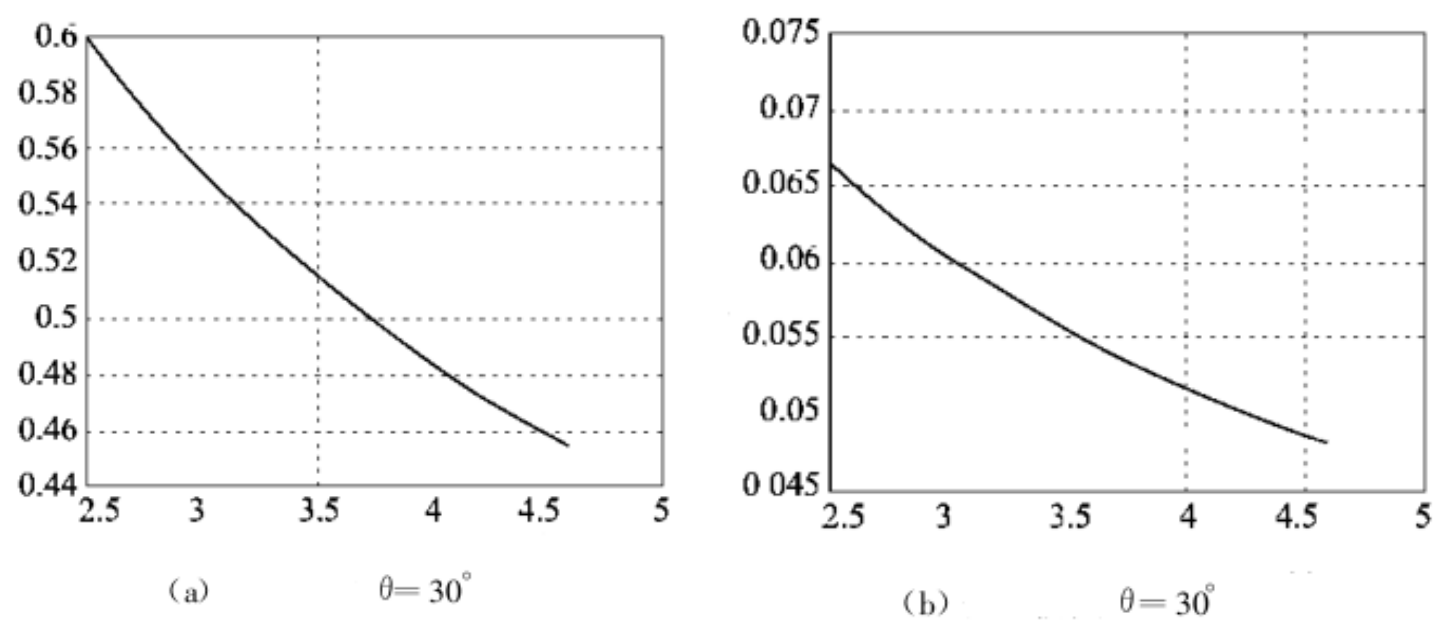

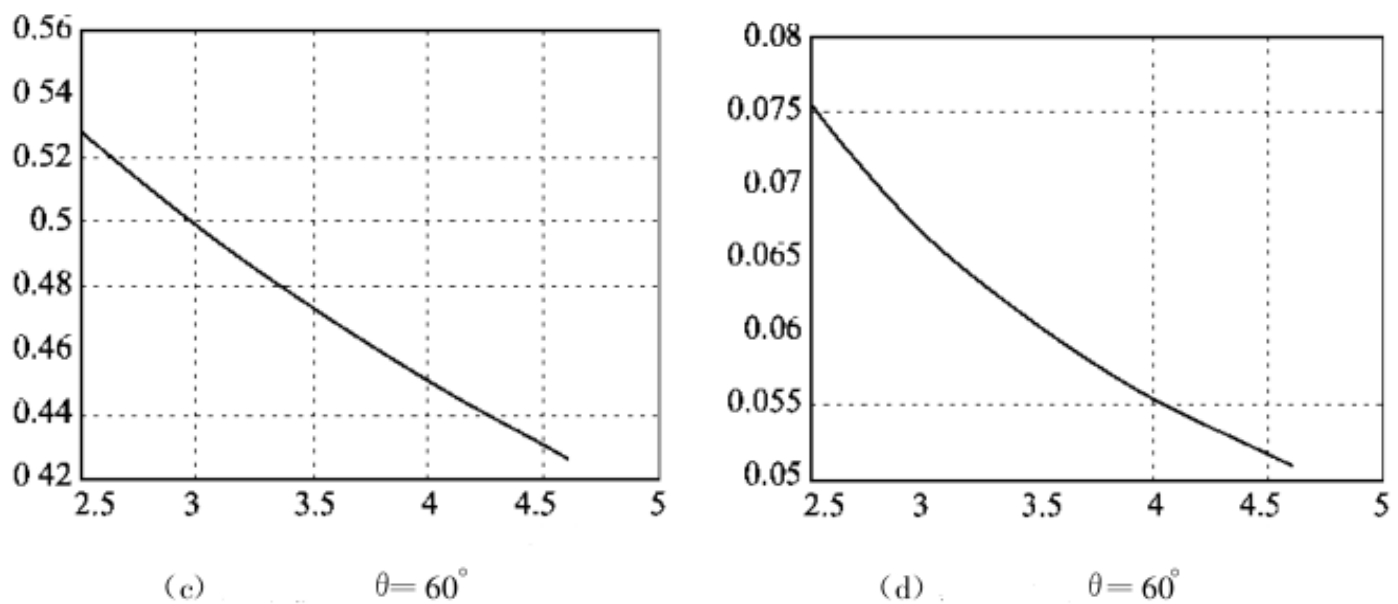

Figure 1: The relationship between the equivalent impedance and the dielectric constant and the incident angle

\section{Wideband Radome Design Method}

In order to meet the requirements of the electrical performance, in the design of the radome can take a single-layer half-wave wall structure, and in order to meet the bandwidth requirements, you can use multi-layer wall Structure to achieve [6]. The type of multi-layer cover wall structure, including A-type sandwich, C-type sandwich and B-type sandwich, etc., and I also used in the design of these types, including A-type interlayer is mainly composed of two relatively close and thin surface layer, such as glass fiber, and low loss, low density and low dielectric constant of the middle core layer, such as honeycomb or foam-like structural materials. The C-type interlayer is mainly formed by the combination of two-layer A-type interlayer. The B-type interlayer is composed of two layers of low-density, low dielectric constant surface layer and compact intermediate core layer [7].

The multi-layer mask wall is a multi-step impedance matching device. The single-step mating device, that is, the larger impedance mutation in the monolayer wall is divided into several small mutations, and the thickness and equivalent characteristics of the abrupt plate Impedance to a reasonable choice, so that the reflection in the entire frequency band is small, in order to be able to follow the relevant design requirements to the antenna envelope for effective design. Under normal circumstances, the commonly used design method is with the frequency change, so that the reflection coefficient can meet the Chebyshev polynomial relations, and become its ladder converter [8]. In the design of Chebyshev's stepped converter, the known condition is that the number of flat plates is $\mathrm{N}$, and the equivalent characteristic impedance ratio of output and input is $\mathrm{r}$, that is, $\mathrm{r}=\mathrm{Z} 0$ $(\mathrm{N}+1) / \mathrm{Z}$ in, where the input and output equivalent impedance are $\mathrm{Z}$.

$\mathrm{Z} 0(\mathrm{~N}+1)$; in a certain operating frequency band, given the maximum power of the reflection coefficient is 2 max. The equivalent electrical thickness of the plates of the layers is equal, ie, D'n is equal, without any loss of the dielectrics of the layers, given the incident angle $\theta 0$ and the polarization direction. It is known that the equivalent impedance is $Z \mathrm{cn}(\mathrm{n}=1,2$, $N)$ in each stratified medium, and the value of $\mathrm{en} 0$ is obtained according to the value of $\mathrm{Z} \mathrm{cn}$. Secondly, it is known that the electrical thickness D'n, according to the value of D'n to find the geometric thickness dn.

The relationship between the reciprocal of the power transfer coefficient and the polynomial of Chebyshev is given as follows:

$$
\frac{1}{|T|^{2}}=1+h^{2} T_{N}^{2}\left(\frac{\cos \varphi}{P}\right)
$$

In which 


$$
\begin{aligned}
& \varphi=\frac{2 \pi d n}{\lambda} \sqrt{\varepsilon_{n 0}-\sin ^{2} \theta_{0}} \\
& h^{2}=\mid \Gamma_{\max }^{2} /\left(1-||_{\max }^{2}\right)
\end{aligned}
$$

The number of half-flat plates is $\mathrm{N}$, and the pending constant associated with the operating band is $\mathrm{P}, 2 \mathrm{max}$. In the given case, the value of $\mathrm{h} 2 \mathrm{can}$ be determined. The first type of Chebyshev polynomial is $\mathrm{T}$ and then the expression of $\mathrm{T} \mathrm{N}(\mathrm{x})$ can be expressed as:

$$
\left\{\begin{array}{l}
T_{0}(x)=1 \\
T_{1}(x)=x \\
T_{2}(x)=2 x^{2}-1 \\
T_{3}(x)=4 x^{3}-3 x \\
T_{4}(x)=8 x^{4}-8 x^{2}+1 \\
\cdots \cdots
\end{array}\right.
$$

\section{Analysis of Broadband Radome Design}

Assuming that the number of radomes is 3 , then $\mathrm{N}=2$, the electromagnetic wave is a vertically polarized wave, and the incident angle $\theta 0$ is equal to 30 degrees, 45 degrees and 60 degrees, $r=0.5$, respectively. (4), (5) and (6), respectively: $\mathrm{r} 1=0.7513, \mathrm{r} 2=0.6746, \mathrm{r} 3=0.5135, \mathrm{r} 4=0.6115, \mathrm{r} 5=0$, $=0.64 ; \varepsilon 20=2.64 ; \varepsilon 30=3.7$; the antenna is the same as the impedance of the antenna The center frequency f $0=7.4 \mathrm{GHz}$, then $\lambda 0=45.1533 \mathrm{~mm}$, calculated,

$$
\begin{aligned}
& |T|^{2}=1-\frac{0.01010101-0.09283329 \cos ^{2} \varphi+}{1.010101-0.09283329 \cos ^{2} \varphi+} \rightarrow \\
& \leftarrow \frac{0.213296 \cos ^{4} \varphi}{0.213296 \cos ^{4} \varphi} \\
& \because \quad \varphi_{=}^{\frac{2 \pi d_{n}^{\prime}}{\lambda}, \quad \mathrm{d}_{n}^{\prime}=\frac{\lambda_{0}}{4}} \\
& \therefore \varphi_{=}^{\frac{\lambda_{0}}{\lambda} \cdot \frac{\pi}{2}=\frac{\mathrm{f}}{f_{0}} \cdot \frac{\pi}{2}}
\end{aligned}
$$

According to the formula (7), it can be calculated that the frequency of the transmission characteristics is 1 to $3 \mathrm{GHz}$ when the antenna angle is 30 degrees, 45 degrees and 60 degrees, and the antenna cover wall is in the case of three layers, the first and second And the dielectric constant and thickness of the third layer are $10=1.54, \mathrm{~d} 1=8 \mathrm{~mm} ; 20=2.40, \mathrm{~d} 2=7 \mathrm{~mm} ; 30=3.48, \mathrm{~d} 3=$ $15 \mathrm{~mm}$. According to the calculated results, $13 \mathrm{GHz}$ case, the power transmission coefficient is more than $85 \%$, indicating that the power transmission characteristics of good [9].

In order to further optimize the wide-band radome, in the course of design and development, the rotary symmetry aeronautical cone-shaped radome is optimized. The antenna is vertically polarized and the actual operating frequency is about $15 \mathrm{GHz}$. The azimuth scanning range is 0 to $60{ }^{\circ}$, composite cover wall structure is anti-static coating and five layers with rain A sandwich structure, different media parameters are controlled as follows. 


\begin{tabular}{lccc}
\hline Layer material $\varepsilon \mathrm{r}$ & $\mathrm{t} \delta$ & $\xi$ Wall thickness range & $(\mathrm{mm})$ \\
\hline 1 Solid glass steel & 4.1 & 0.016 & $0.1--1.0$ \\
2 Honeycomb core material & 1.0 & 0.005 & $1.0--20$ \\
3 Solid glass steel & 4.0 & 0.017 & $0.1--1.0$ \\
4Rust-proof coating & 3.4 & 0.060 & $0.1--0.5$ \\
5Anti-static coating & 6.5 & 0.500 & $0.01--0.05$ \\
\hline
\end{tabular}

In the above table, the optimization of the single target for different parameters is carried out. In the actual radome design, different parameters are expected to achieve the optimal target at the same time. If the single objective optimization model is expanded, it can be closely integrated with the multi-objective optimization method up, but also to quickly handle the radome design during the multi-objective optimization, global optimization and a series of problems.

\section{Conclusion}

Based on the theoretical basis of the transmission line, this paper analyzes the design principle of the broadband radome, and provides the reference for the design of the broadband radome through the calculation and design of the broadband radome.

\section{References}

[1] Wang Yang-zhi, Zhang Lin-xi, Wei Gao.Design of New Broadband Microstrip Antenna Based on HFSS[J]. Journal of System Simulation, 2007, 11: 2603-2606.

[2] Lei Jingxuan, Shi Zhiwei, Hu Wei, Zhao Zhongjian, Wu Hao, Tan Qiao. Research progress of high temperature and wide band ceramic missile radome[J]. Chinese Journal of Ceramics, 2007, 02: 144-149.

[3] Zhu Yu-xiao, Zhong Shun-shi, Xu Sai-qing, Zhang Li-na.Design of miniaturized planar helical antenna and its wideband Balun[J]. Journal of Shanghai University (Natural Science Edition), 2008, 06: $581-584$

[4] Chen Jian-jun, Song Xue-rui, Cao Hong-han.Design of Wideband High Gain RFID Microstrip Antenna Array [J]. Microcomputer and Application, 2012,10: 58-60 + 64

[5] Jia Lei, LI Gao-sheng, Cao Qun-sheng, Guo Xu.Study on Electrical Performance Test of a Type of Airborne Broadband Radome [J]. Acta Mathematica Sinica, 2012, 04: 48-51 + 56.

[6] Liu Jianxia, Sun Xiaoling, Xi Xinxin. Design and simulation of a new broadband microstrip antenna[J]. Journal of Taiyuan University of Technology, 2010, 03: 248-251.

[7] Gu Wei, Zhang Pu-pu, Zhong Shun-shi.Multi-type, wideband rectangular microstrip antenna with organic magnetic material as substrate[J]. Journal of Shanghai University (Natural Science Edition), 2002, 01: 25-27

[8] Li Hongbin, Ding Weiping, Yu Tongbin, Cao Wenquan. Design and fabrication of a wideband circularly polarized microstrip antenna with beam broadening [J]. Military Communications Technology, 2011, 04: 71-73 + 82 .

[9] Zhou Tian, Yue Yan - tao, Xu Guan - xiong, Zhang Yang - yang, JI Chun - lin. Electroelectric optimization design in ultra - wideband communication transmission [J]. Journal of Science and Electronics, Hohhot, 2015, 01: 76-79. 